\title{
Design and Analysis of a Bearingless Fixed-pole Rotor Induction Motor
}

\author{
Xiaoting Ye, and Zebin Yang \\ School of Electrical and Information Engineering \\ Jiangsu University \\ Zhenjiang, China \\ xiaotingye@163.com, zbyang@ujs.edu.cn
}

\author{
Jianguo Zhu, and Youguang Guo \\ School of Electrical, Mechanical and Mechatronic Systems \\ University of Technology Sydney \\ Sydney, Australia \\ Jianguo.Zhu@uts.edu.au, Youguang.Guo-1@uts.edu.au
}

\begin{abstract}
Both torque winding magnetic field and suspension winding magnetic field induce currents in the rotor in conventional bearingless induction motor (BIM). Owing to the induced currents of suspension winding magnetic field, the airgap magnetic field is affected that can result in the error of radial suspension forces. To overcome such drawbacks, a novel BIM with fixed-pole rotor is proposed, which is called bearingless fixed-pole rotor induction (BFPRI) motor. First, the structure of BFPRI motor is designed and introduced. Then, rotor currents and radial suspension forces are calculated and compared with conventional BIM. Finally, the prototype motor equipped with fixed-pole rotor is constructed. In this novel motor, only the torque winding magnetic field will induce currents in the rotor, which makes the precision of radial suspension forces higher and reduces the complexity of BIM control system. Analytical results are validated through finite element analysis (FEA) and experimentation.
\end{abstract}

Keywords-bearingless induction motor; fixed-pole rotor; operation principle; rotor currents; radial suspension force

\section{INTRODUCTION}

BIM possesses the advantages of simple structure, small torque ripple, low noise, easy magnetic weakening and high reliability, especially the squirrel cage rotor with low cost, high mechanical strength and easy maintenance [1], [2]. However, such a squirrel cage rotor BIM has inherent technical problems that both torque winding magnetic field and suspension winding magnetic field induce rotor currents. For a small motor with a low speed, the induced currents of suspension winding are less influenced. When calculating the radial suspension forces, the currents influence in this part is negligible. However, for high-speed motors with large power, the influence is apparent. As a consequence, the actual airgap rotating magnetic field has a larger difference in amplitude and phase compared with the airgap rotating magnetic field that ignores this part of currents effect. The errors will be brought to radial suspension forces, which further make the radial suspension forces coupled and the phase margin of suspension system smaller, resulting in system oscillation. To solve these problems, BIM with fixed-pole rotor, namely, bearingless fixed-pole rotor induction (BFPRI) motor, has been proposed. BFPRI motor not only has the advantages of traditional BIM, but also provides good suspension characteristics due to the special design of the rotor.

\section{MOTOR TOPOLOGIES AND PRINCIPLE}

For the verification of the proposed BFPRI motors, two 3D-FEA models with the same stators, turns of torque winding and suspension winding and electric loading are established. As shown in Fig. 1, the BFPRI motor adopts a special rotor design structure which has 11 sets of fixed-pole rotor bars insulated from each other. Single fixed-pole rotor bar is composed of two end rings and two bars. Two end rings and bars are integrated structure. When 2-pole rotating magnetic field is generated by the torque winding, EMFs and currents are induced in the rotor. On the contrast, if 4-pole rotating magnetic field is applied, the sum of the induced EMFs are zero in the rotor because of the cancellation effect, and consequently 4-pole rotating magnetic field will not induce currents in the rotor. Therefore, fixed-pole rotor structure solves the problem of the effect of suspension winding currents on the radial suspension forces. The BFPRI motor has excellent suspension performance.

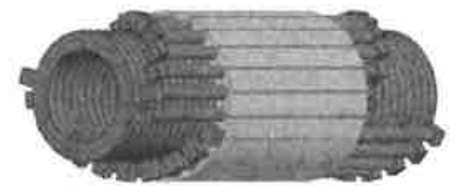

Figure 1. Fixed-pole rotor of BFPRI motor

\section{Electromagnetic Performance Comparsion}

\section{A. Rotor currents}

For the comparison of the rotor induced currents under different stator winding magnetic fields, three different situations in two types of motors are analyzed through FEA, which are shown in Fig. 2. First, based on the excitation of torque winding, the rotor induced currents are shown in Fig. 2(a). The rotor currents waveform for BFPRI motor almost coincides with that of conventional BIM. Second, if only the suspension winding applied, the rotor currents are shown in Fig. 2(b). It can be seen that the amplitude of induced currents is almost zero in the BFPRI motor, while the induced currents 
amplitude changes from $-250 \mathrm{~A}$ to $150 \mathrm{~A}$ in conventional BIM, by which the airgap magnetic field will be seriously influenced. Third, under the condition of the two sets of stator winding application, as shown in Fig. 2 (c), the effect of the rotor currents induced by suspension winding magnetic field in conventional BIM leads to waveform distortion of the rotor currents. In BFPRI motor, the waveform of the rotor currents under two magnetic fields is almost the same with the rotor currents under torque winding magnetic field only, which means suspension winding excitation has no influence on the airgap magnetic field.

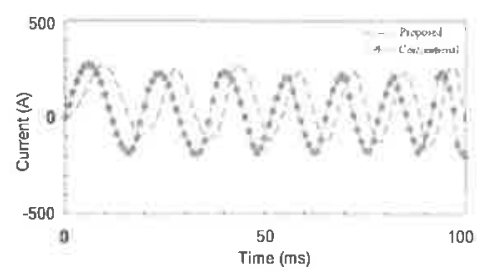

(a)

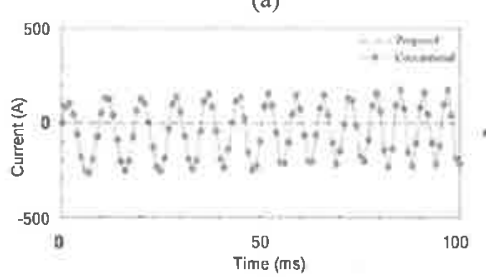

(b)

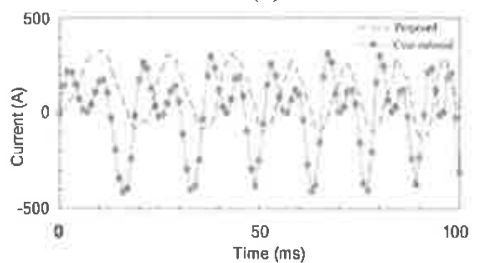

(c)

Figure 2. Rotor currents waveforms of conventional BIM and BFPRI motor (a) Excited by torque winding (b) Excited by suspension winding (c) Excited by two scts of windings

\section{B. Radial suspension Force}

Fig. 3 shows the waveforms of the computed radial suspension forces. The average value of radial suspension forces in conventional BIM and in BFPRI motor are approximately $170 \mathrm{~N}$ and $420 \mathrm{~N}$, respectively. Compare of the finite element waveform curves of suspension forces, the waveforms of conventional BIM is distorted and the resultant forces are instable with a large fluctuation, due to the influence of induced current of suspension winding magnetic field. The waveforms of $F_{\mathrm{x}}$ and $F_{\mathrm{y}}$ in BFPRI motor approach to sine wave, and the stability and amplitude of resultant forces $F_{\mathrm{m}}$ are higher.

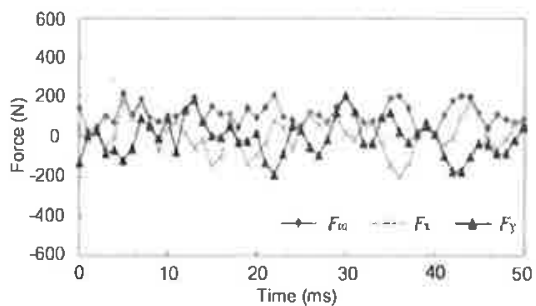

(a)

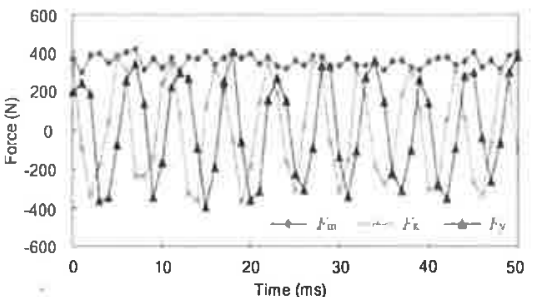

(b)

Figure 3. Radial suspension forces waveforms (a) Conventional BIM (b) BFPRI motor

\section{EXPERIMENTAL RESULTS}

To demonstrate the effectiveness of the proposed BFPRI motor, the experimental research on the digital control system platform of the BFPRI motor is carried out. The same contro system and experimental platform are used in conventional BIM and BFPRI motor. Fig. 4 shows the radial displacements in $x$-axis and $y$-axis direction and the orbit of the rotor center in conventional BIM and BFPRI motor. According to the experiment results, it can be found clearly that the maximum deviation of the radial displacements of the rotor centroid in $x$ axis and $y$-axis direction is decreased, and the peak-to-peak value of the radial displacement of the rotor in $x$-axis and $y$-axis direction is reduced.

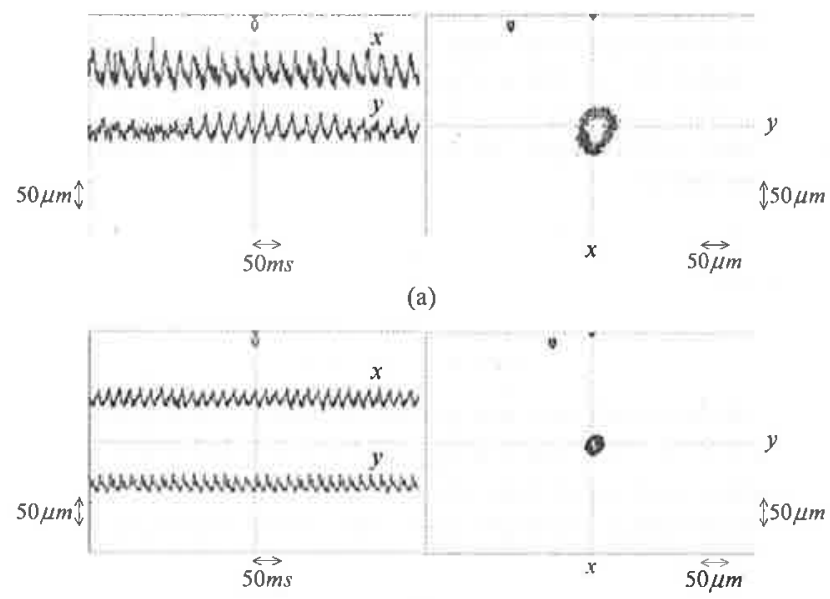

(b)

Figure 4. Expcrimental result of rotor radial displacement and rotor trajectory (a) conventional BIM (b) BFPRI motor

From the comparisons of the results from FEA simulation and experiment results, the special design of fixed-pole rotor in BFPRI motor compensate for radial suspension forces errors caused by the interference of suspension winding currents to the airgap magnetic field, and the merits of fixed-pole rotor are helpful for BIM design and performance optimization.

\section{REFERENCES}

[1] X Sun, L Chen, and Z Yang. "Overvicw of bearingless induction motors," Math. Problems Eng., pp. 1-10, 2014.

[2] W Bu, Z Li, C Lu, X Wang, J Xjao, "Rescarch on the least square support vector machine displacement observer of a bearingless induction motor," Trans. Inst. Mcas. Control, vol.10, no.1, pp.1-12, 2015. 